\section{Retinal and choroidal thickness evaluation by SD-OCT in adults with obstructive sleep apnea-hypopnea syndrome (OSAS)}

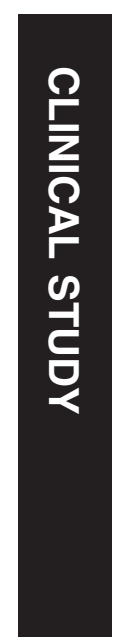

${ }^{1}$ Department of

Ophthalmology, Beijing Anzhen Hospital, Capital Medical University, Beijing, China

${ }^{2}$ Department of Respiratory Medicine, Beijing Anzhen Hospital, Capital Medical University, Beijing, China

${ }^{3}$ Beijing Institute of Ophthalmology, Beijing Tongren Eye Center, Beijing Tongren Hospital, Capital Medical University, Beijing, China

Correspondence:

$X$ Peng, Beijing Institute of Ophthalmology, Beijing Tongren Eye Center, Beijing Tongren Hospital, Capital Medical University, 17

Hougou Lane, Chong Nei Street, Dongcheng district, Beijing 10005, China.

Tel: + 86 13691287978;

Fax: + 8601058268243.

E-mail: drpengxiaoyan@

sina.com

Obstructive sleep apnea-hypopnea syndrome (OSAS) is the most common type of sleepdisordered breathing and is characterized by recurrent episodes of upper airway collapse and paroxysmal anoxia during sleep accompanied
Received: 12 July 2013 Accepted in revised form: 25 November 2013 Published online: 10 January 2014 
by arousal from sleep and daytime sleepiness.

The alternation between hypoxia and arousal can stimulate the sympathetic nervous system, causing a myriad of downstream effects. ${ }^{1}$ Meanwhile, hypoxia can lead to increased blood pressure and a weakened response of the vascular system to vasodilatation factors, resulting in dysfunction of blood vessel and tissue selfregulation ${ }^{2}$ and vascular endothelial disorders. ${ }^{3}$ Previous studies have suggested that OSAS acts as an independent risk factor and leads to hypertension, heart disease, and stroke through the mechanisms described above. ${ }^{4-6}$

OSAS can have a similar effect on the eye. According to some reports, the prevalence of primary open-angle glaucoma is increased in patients with OSAS. ${ }^{7,8}$ Further, examination by perimetry revealed that the retinal nerve fiber layer of some OSAS patients with a normal visual field and no obvious optic neuropathy tended to be thin, ${ }^{9}$ indicating that OSAS may affect the physiological structure of the optic nerve.

The retina has the greatest oxygen demand in darkness, especially at night. ${ }^{10}$ The vascular supply to the outer part of the retina is through the choroid and that to the inner part is from the retinal vessels. Choroidal vessels are among the tissues with the fastest blood flow and are innervated by a large number of sympathetic nerves, ${ }^{11}$ but they lack autonomic innervation; in contrast, retinal vessels have slow blood flow and large oxygen intake, and therefore the inner part of the retina is sensitive to hypoxia. ${ }^{12}$ West et al ${ }^{13}$ reported that the incidence of macular edema is twofold higher in diabetic patients with OSAS compared with patients with simple diabetes, and that OSAS is one of the independent risk factors of diabetic retinopathy in diabetics. Meanwhile, the incidence of proliferative diabetic retinopathy has increased significantly in diabetic patients who also have OSAS. ${ }^{14}$ OSAS may promote vascular growth factor release by fluctuations in blood pressure and oxygen and activation of the sympathetic nervous system, thus aggravating the damage of diabetes to retinal blood vessels. However, research is lacking regarding the effect of OSAS on macular retinal and choroidal tissue structures in individuals without basic retinopathy.

Spectral domain-optical coherence tomography, which is a relatively new instrument for clinical in vivo observation and retinal tissue follow-up evaluations, can execute repeated, high-resolution cross-sectional scans of the retina. The examination is more quantitative than an ophthalmoscope examination, and even small changes in the retina can be detected. In addition, the choroidal thickness can be measured by a choroidal enhancement model.

The purpose of this study was to assess the macular retinal and choroidal thickness changes in OSAS patients with no significant symptoms and pathological changes in fundus by spectral domain-optical coherence tomography.

\section{Materials and methods}

We consecutively recruited 74 patients with OSAS who were transferred from the sleep monitoring center to the otolaryngology department. Nine patients were excluded for the following reasons: history of eye surgery (2), mature cataract that prevented ocular examination (1), hypermyopia (2), and diabetes (4). Ophthalmic fundoscopy did not reveal retinopathy in the remaining 65 patients. The Medical Ethics Committee of the Beijing Anzhen hospital approved the study protocol. All participants provided written informed consent according to the Declaration of Helsinki.

\section{Polysomnography (PSG)}

Every patient was monitored using a portable sleep monitor (Embletta, PDS model 2601-1 × 10Xact Trace/ Embletta, Broomfield, CO, USA); all patients were reminded of the precautions to be taken during the sleep monitoring process by the same technician in the afternoon before the patient underwent sleep monitoring. All monitoring results were scored by the same experienced technicians who were blinded to the basic characteristics of the patients. The apnea/hypopnea index (AHI) of the patients was evaluated using the American Academy of Sleep Medicine (AASM) standards. ${ }^{15}$ The patients were diagnosed and classified according to the AHI as follows: those without OSAS $(\mathrm{AHI} \leq 5)$; those with mild OSAS (AHI 5-15); those with moderate OSAS (AHI 15-30); and those with severe OSAS (AHI $\geq 30$ ). The following variables were assessed: the average oxygen saturation $\left(\mathrm{MSaO}_{2}\right)$, which reflects the average blood oxygen status of the patient at night; the oxygen desaturation index (ODI), which reflects the frequency with which the arterial oxygen saturation falls more than $4 \%$ in $1 \mathrm{~h}$; and the TS90\%, which reflects the percentage of time that the arterial oxygen saturation is less than $90 \%$ of the total sleep time.

Every patient underwent a comprehensive ophthalmologic examination, including best-corrected visual acuity, reaction of pupil to light, slit-lamp examination, Goldmann applanation tonometry, and mydriatic fundus photography. The values from the left eye were selected for statistical analysis.

\section{Optical coherence tomography (OCT)}

An OCT scan was performed on every patient from 9a.m. to 12a.m. after mydriasis using Mydrin-P. The macular 
retinal thickness was measured using three-dimensional (3D) OCT-1000 (Topcon, Tokyo, Japan) in an internally installed, posterior macular horizontal linear scan mode, which automatically drew the macular area topographic map and selected the central circle, which is within $0.5 \mathrm{~mm}$ to the fovea, and four quadrants (superior, nasal, inferior, and temporal) within $1.5 \mathrm{~mm}$ to the fovea outside the central circle to record macular thickness. We measured the choroidal thickness of the subfovea and $1 \mathrm{~mm}$ nasal and temporal to the macular central fovea using an in-built, enhanced choroidal landscape scan. The choroidal thickness was defined as the vertical distance between the retinal pigment epithelium (RPE) high-reflective tape and the internal scleral boundary (Figure 1 choroidal thickness measured by OCT). We considered the average values of three different measurements taken per week by the same doctor, who was unaware of the patients' OSAS status. The OCT images obtained from all patients did not have obvious macular edema or vitreous macular lesions.

\section{Statistical analysis}

Data analysis was conducted using SPSS software version 19.0 (SPSS, Inc., Chicago, IL, USA). Continuous

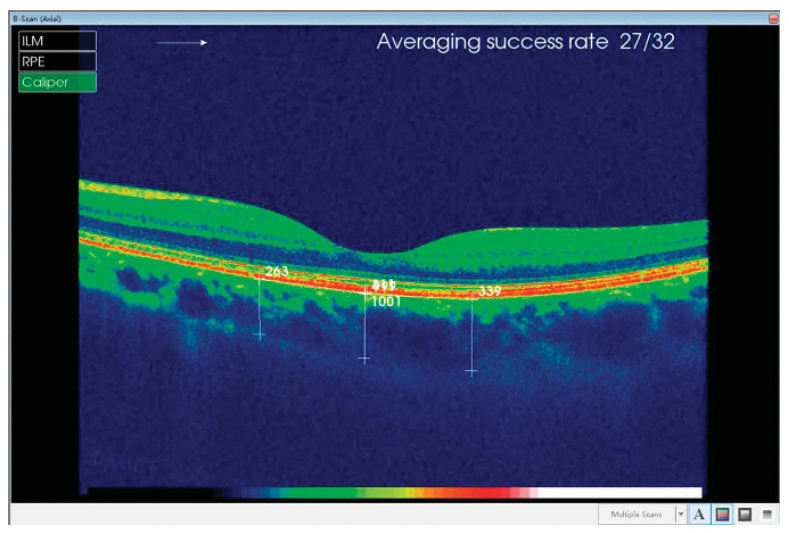

Figure 1 Choroidal thickness measured by OCT. measurement values are presented as mean \pm standard deviation (SD) and expressed as percentages. The differences in measurement values between groups were determined using one-way analysis of variance (ANOVA) and the differences between subgroups were analyzed with the LSD test. Categorical data were studied using the $\chi^{2}$ test. Age and diopter values were corrected using covariance analysis before comparing choroidal thickness between the groups. Multiple stepwise regression analysis was performed to correlate retinal and choroidal thickness to significant ophthalmologic variables such as age, diopter, and PSG index $\left(\mathrm{MSaO}_{2}, \mathrm{ODI}, \mathrm{TS} 90 \%\right)$. The macular thickness in all groups was compared using nonparametric tests, when the outliers were excluded. $P$-values $<0.05$ were considered statistically significant. Then, the macular thickness between each of two groups was also analyzed with nonparametric tests combined with the Bonferroni test. $P$-values $<0.0083$ were considered statistically significant.

\section{Results}

All 53 OSAS patients included in the study were divided into four groups according to the AHI (measured by using the PSG test): control group, 12 patients (AHI $\leq 5$ ); mild group, 13 patients (AHI 5-15); moderate group, 15 patients (AHI 15-30); and severe group, 25 patients (AHI $\geq 30$ ). The basic characteristics and OSAS-related index of the patients are shown in Table 1. No significant difference in age, sex ratio, intraocular pressure, and diopter was found between the groups.

The medium macular thickness in the center within $0.5 \mathrm{~mm}$ to the fovea and that of the four quadrants outside the center (superior, nasal, inferior, and temporal) within $1.5 \mathrm{~mm}$ to the fovea are shown in Table 2 and Supplementary Information. Foveal thickness between two groups revealed no significant difference between the control and mild group $(P=1.000)$, nor between the control and moderate group

Table 1 The basic characteristics and OSAS-related index of the patients

\begin{tabular}{|c|c|c|c|c|c|}
\hline & Control & Mild & Moderate & Severe & P-value \\
\hline Eye $(n)$ & 12 & 13 & 15 & 25 & \\
\hline Age (years) & $51.4 \pm 8.2$ & $56.7 \pm 12.2$ & $55.9 \pm 9.2$ & $52.4 \pm 11.9$ & 0.481 \\
\hline Male (\%) & 66.7 & 67.2 & 73.3 & 84 & 0.446 \\
\hline IOP (mm Hg) & $17.3 \pm 2.3$ & $15.5 \pm 2.4$ & $16.1 \pm 3.0$ & $17.1 \pm 2.8$ & 0.264 \\
\hline Diopter (D) & $0.21 \pm 1.07$ & $-0.39 \pm 1.19$ & $-0.37 \pm 1.28$ & $-0.16 \pm 1.31$ & 0.851 \\
\hline $\operatorname{HBP}(\%)$ & 50.0 & 46.2 & 62.0 & 54.2 & 0.229 \\
\hline $\mathrm{AHI}$ & $1.4 \pm 1.0$ & $9.8 \pm 3.0$ & $21.6 \pm 5.2$ & $50.2 \pm 16.2$ & $<0.001^{* *}$ \\
\hline $\mathrm{MSaO}_{2}(\%)$ & $97.8 \pm 0.5$ & $95.4 \pm 1.2$ & $95.3 \pm 1.4$ & $92.5 \pm 2.5$ & $<0.001^{* *}$ \\
\hline BMI $\left(\mathrm{kg} / \mathrm{m}^{2}\right)$ & $25.3 \pm 4.1$ & $29.7 \pm 3.6$ & $27.2 \pm 3.9$ & $29.9 \pm 3.5$ & $0.03^{*}$ \\
\hline
\end{tabular}

Abbreviations: AHI, apnea/hypopnea index; BMI, body mass index; HBP, hypertension; IOP, intraocular pressure; $\mathrm{MSaO}_{2}$, average oxygen saturation. ${ }^{* *} P<0.01,{ }^{*} P<0.05$. 
$(P=0.428)$. However, the foveal thickness of the control group was significantly thinner than that of the severe group $(P=0.000)$. Similarly, no significant difference in nasal macular thickness was found between the control group and the mild group $(P=0.343)$, nor between the control and moderate group $(P=0.015)$; whereas the nasal macular thickness of the control group was significantly thinner than that of the severe group $(P=0.008)$. The superior, inferior, and temporal macular thicknesses were not significantly different between the groups ( $P=0.096,0.056$, and 0.182 , respectively). When evaluating age, diopter, and PSG index $\left(\mathrm{MSaO}_{2}, \mathrm{ODI}\right.$, TS90\%) by using multiple stepwise regression analysis, we found a significant correlation between ODI and macular center thickness $\left(r=0.357, R^{2}=0.127, P=0.004\right)$, with an ODI coefficient of $0.457(P=0.004)$. Similarly, a significant correlation was found between ODI and nasal macular thickness $\left(r=0.265, R^{2}=0.070, P=0.033\right)$, with an ODI coefficient of $0.233(P=0.033)$.

The subfoveal choroidal thickness of OSAS patients was $233 \pm 56 \mu \mathrm{m}$, the choroidal thickness $1 \mathrm{~mm}$ nasal to the macular central fovea was $211 \pm 42 \mu \mathrm{m}$, and the choroidal thickness $1 \mathrm{~mm}$ temporal to the macular central fovea was $278 \pm 36 \mu \mathrm{m}$. The difference between these three areas was statistically significant $(P=0.043)$. In the control group, the subfoveal choroidal thickness was significantly thicker than that of $1 \mathrm{~mm}$ nasal to the macular central fovea $(P=0.017)$, whereas no significant difference was observed with that of $1 \mathrm{~mm}$ temporal to the macular central fovea $(P=0.564)$.

The values of the subfoveal choroidal thickness and that of $1 \mathrm{~mm}$ nasal and temporal to the fovea are shown in Table 3. After correcting for age and diopter, the subfoveal choroidal thickness was found to be significantly different between the groups $(F=3.657$, $P=0.017)$. A pairwise comparison between the groups revealed that the subfoveal choroidal thickness of the severe group was significantly thinner than that of the control group, mild group, and moderate group ( $P=0.023,0.006$, and 0.036 , respectively). However, no significant differences were found between the other groups $\left(P_{\text {control\&mild }}=0.703, P_{\text {control\&moderate }}=0.762\right.$, and $\left.P_{\text {mild\&moderate }}=0.477\right)$. Further, the choroidal thickness $1 \mathrm{~mm}$ nasal to the fovea was significantly different between the groups after correcting for age and diopter $(F=3.411, P=0.023)$. A pairwise comparison between the groups showed that the choroidal thickness $1 \mathrm{~mm}$ nasal to the fovea was significantly thinner in the severe group compared with the control group and mild group ( $P=0.013$ and 0.010 , respectively), whereas no significant difference was observed with that in the moderate group. Significant differences were not found between the other groups $\left(P_{\text {control\&mild }}=0.963, P_{\text {control\&moderate }}=0.299\right.$, $P_{\text {mild\&moderate }}=0.260$ ). In contrast, no significant
Table 2 The medium of the macular thickness $(\mu \mathrm{m})$

\begin{tabular}{lccccl}
\hline & Control & Mild & Moderate & Severe & P-value \\
\hline Central & 231 & 233 & 251 & 245 & $0.001^{* *}$ \\
Superior & 301 & 299 & 312 & 313 & 0.096 \\
Nasal & 297 & 299 & 315 & 309 & $0.016^{*}$ \\
Inferior & 289 & 282 & 304 & 294 & 0.056 \\
Tempor & 290 & 284 & 300 & 294 & 0.182 \\
\hline
\end{tabular}

${ }^{*} P<0.05,{ }^{* *} P<0.01$

Table 3 Choroidal thickness at different positions $(\mu \mathrm{m})$ in patients with various degrees of OSAS

\begin{tabular}{llllll}
\hline & Control & Mild & Moderate & Severe & P-value \\
\hline Subfovea & $254 \pm 32$ & $260 \pm 48$ & $242 \pm 50$ & $213 \pm 58$ & $0.024^{*}$ \\
Nasal & $237 \pm 27$ & $230 \pm 38$ & $212 \pm 35$ & $201 \pm 46$ & $0.038^{*}$ \\
Temporal & $243 \pm 38$ & $239 \pm 30$ & $233 \pm 31$ & $219 \pm 40$ & 0.186 \\
\hline
\end{tabular}

${ }^{*} P<0.05$.

difference in the choroidal thickness $1 \mathrm{~mm}$ temporal to the fovea was found between the groups ( $F=1.994$, $P=0.132$ ). Multiple stepwise regression analysis on the subfovea choroidal thickness and significant ophthalmologic variables such as age, diopter, and PSG index $\left(\mathrm{MSaO}_{2}, \mathrm{ODI}, \mathrm{TS} 90 \%\right)$ showed that the choroidal thickness was significantly correlated with diopter ( $\left.r=0.520, R^{2}=0.270, P<0.001\right)$, with a coefficient of 0.327 $(P<0.001)$, and with ODI $\left(r=0.520, R^{2}=0.165, P=0.001\right)$, with a coefficient of $-0.370(P=0.001)$.

\section{Discussion}

The choroid contains ample amounts of blood vessels and pigments, which provide nutrients to the outer retina, and its normal structure and function provide the basis for maintaining normal retinal function. The normal choroid was thickest underneath the fovea and was thinner in the surrounding area, which was especially obvious in the nasal direction. This finding may be because the photoreceptor is most active at the macular center concave, where it has no direct retinal blood supply; thus, the choroid is thickest underneath the fovea because it must provide adequate blood supply. ${ }^{16-18}$ In the Chinese population, the subfoveal choroidal thickness is not correlated with age in subjects younger than 60 years, whereas the subfoveal choroidal thickness is relatively thinner and significantly negatively correlated with age in the population older than 60 years. ${ }^{18}$ Meanwhile, the choroidal thickness was shown to increase along with the severity of the diopter, ${ }^{19}$ and a pattern of diurnal variation exists. ${ }^{20}$ Therefore, we carried out all of the choroidal and retinal 
OCT examinations from 9a.m. to 12a.m. in the present study. Our statistical analysis showed that the subfoveal choroidal thickness was not correlated with age, which may be due to the relative concentration in age of the subjects. In addition, the subfoveal choroidal thickness decreased only by $15.6 \mu \mathrm{m}$ for each decade of life, ${ }^{16}$ indicating that the choroidal thickness change caused by age is not significant compared with diopter and ODI. Consistent with the results of Sogawa et al, ${ }^{19}$ increased choroidal thickness was associated with increased diopter. The subfoveal choroidal thickness was significantly thinner in the severe group after correcting for the diopter, and the degree was associated with ODI, indicating that the degree of thinning of the choroid is associated with fluctuation in the frequency of $\mathrm{SaO}_{2}$. Thus, we conclude that alternating hypoxia and arousal can stimulate the sympathetic nervous system, causing a myriad of downstream effects, ${ }^{1}$ including the induction of structural changes in choroidal blood vessels through the sympathetic nerves ${ }^{21}$ and inner nerves ${ }^{22}$ distributed in the choroidal blood vessels. Choroidal blood flow is not vulnerable to the effects of transient $\mathrm{SaO}_{2}$ changes because of the rapid choroidal blood flow and small arteriovenous oxygen partial pressure change, ${ }^{23}$ however, it is sensitive to hypercapnia because the increase in choroidal blood flow is $\sim 1.5 \%$ per $1 \mathrm{~mm} \mathrm{Hg}$ increase in $\mathrm{PCO}_{2}{ }^{24}$ Meanwhile, hypoxia stimulates the activity of hypoxia-inducible factor ${ }^{25}$ and increases the expression of vascular growth factors (such as vascular endothelial growth factor); ${ }^{26}$ these activities are followed by destruction of the vascular endothelium, changes in vascular permeability, and a subsequent decrease in choroidal thickness.

The retina, which is one of the most metabolically active tissues in the human body, ${ }^{10}$ has a double blood supply from the central artery and the choroid. The photoreceptors, which are the main components of the outer layers of the retina, are mainly supplied by the choroid and have an extremely high oxygen consumption. The inner retina is supplied by retinal blood vessels and is sensitive to tissue oxygen partial pressure changes; thus, transient anoxia may result in an immediately increased blood flow to the retina, and a subsequent return to normal flow. ${ }^{12}$ However, long-term low blood oxygen can lead to a significant increase in retinal blood flow. ${ }^{27}$ Because the oxygen supply to the outer retina is dependent upon the oxygen partial pressure difference between the choroid and the retina, decreased choroidal blood oxygen partial pressure may reduce the pressure difference between the choroid and the retina, further aggravating retinal hypoxia. ${ }^{28}$ In the absence of autoregulation the choroid is more vulnerable to systemic risk factors rather than to local ones, such as central serous chorioretinopathy. ${ }^{29}$ Under stress, in the presence of sympathomimetic agent use, and in the event of autonomic dysfunction, which triggers CSC, the choroid was thickened, which increased the hydrostatic pressure within the choroid, leading to PED formation. Meanwhile, a thinner choroidal thickness and a reduced blood flow could further aggravate retinal hypoxia. As stated above, hypoxia stimulates the activity of the hypoxia-inducible factor, ${ }^{25}$ increases the expression of vascular growth factors (such as vascular endothelial growth factor), ${ }^{26}$ and results in pathological changes to the retinal vessel. Long-term abnormal blood flow and hypoxia can lead to retinal layer cell damage, cellular edema, pigment epithelium damage, blood-retinal barrier breakdown, and an increase in choroidal exudation to the retina. Because the photoreceptors in the macular fovea have the greatest activity and have the highest oxygen consumption, they are most likely to be involved, resulting in obvious thickening of the retina in the macular fovea.

This change may be similar to the change that occurs in diabetes. The vascular lesions of diabetes are caused by high blood glucose, in which long-term choroidal and retinal hypoxia lead to choroidal thinning and retinal thickening. ${ }^{30}$ The photoreceptors in the macular center fovea have the highest oxygen consumption and its blood supply is derived completely from the choroid; thus, changes in the choroid and retina in the macular center fovea are more obvious compared with other areas because the influence of the ischemia hypoxia state is more obvious in this region. Mason et al ${ }^{31}$ found that patients with clinically significant diabetic macular edema have a high prevalence of OSAS, and treatment with continuous positive airway pressure might improve macular edema. ${ }^{32}$ This confirmed that the intermittent hypoxia state in diabetic patients with OSAS can aggravate the vascular damage on the structure of the macular retina and choroid caused by diabetes, leading to increased macular vascular permeability and aggravation of macular edema. Local macular metabolism and vascular function can be restored following an improvement in the hypoxic condition, with subsequent alleviation of macular edema.

Our results differ from those of Casas et al, ${ }^{9}$ who found that only the temporal macular retinal thickness was significantly higher in patients with mild-to-moderate OSAS compared with those with severe OSAS, whereas the macular thickness of the other areas was not significantly different in any group. Casas et al analyzed both eyes of 50 OSAS patientss, but we selected only one eye for each of our subjects. Further, regarding the diagnostic criteria, in contrast to our use of the standard value (AHI >5), Casas et al used AHI > 4 to diagnose patients with an OSAS. For control group selection, we selected OSAS patients diagnosed by PSG, whereas 
Casas et al selected age-matched patients without PSG determination, which may have resulted in missed diagnoses.

In summary, we propose that a sympathetic nervous regulation disorder exists in OSAS patients because of chronic intermittent hypoxia, which is followed by changes in the structure of the normal retina and choroid. This is especially true for diabetic patients with an abnormal blood vessel function, in which OSAS may further aggravate the retinal and choroidal damage.

The main drawbacks of this study are the small cohort and the fact that all subjects were undergoing sleep monitoring, which introduced selection bias. Thus, our results do not represent OSAS patients in general. Finally, we were unable to obtain exact information regarding the duration of the OSAS, which has a certain influence on the degree of hypoxia.

\section{Summary}

What was known before

- OSAS may affect the eyes

What this study adds

- OSAS patients showed decreased foveal and nasal macular thickness, similar to that in the subfoveal and nasal choroidal thickness

\section{Conflict of interest}

The authors declare no conflict of interest.

\section{References}

1 Fletcher EC. Sympathetic overactivity in the etiology of hypertension of obstructive sleep apnea. Sleep 2003; 26: 15-19.

2 Kato M, Roberts-Thomson P, Phillips BG, Haynes WG, Winnicki M, Accurso V et al. Impairment of endotheliumdependent vasodilation of resistance vessels in patients with obstructive sleep apnea. Circulation 2000; 102: 2607-2610.

3 Priou P, Gagnadoux F, Tesse A, Mastronardi ML, Agouni A, Meslier $\mathrm{N}$ et al. Endothelial dysfunction and circulating microparticles from patients with obstructive sleep apnea. Am J Pathol 2010; 177: 974-983.

4 Parish JM, Somers VK. Obstructive sleep apnea and cardiovascular disease. Mayo Clin Proc 2004; 79: 1036-1046.

5 Lattimore JD, Celermajer DS, Wilcox I. Obstructive sleep apnea and cardiovascular disease. J Am Coll Cardiol 2003; 41: 1429-1437.

6 Yaggi HK, Concato J, Kernan WN, Lichtman JH, Brass LM, Mohsenin V. Obstructive sleep apnea as a risk factor for stroke and death. N Engl J Med 2005; 353: 2034-2041.

7 Bendel RE, Kaplan J, Heckman M, Fredrickson PA, Lin SC. Prevalence of glaucoma in patients with obstructive sleep apnoea - a cross-sectional case series. Eye 2008; 22: 1105-1109.
8 Lin PW, Friedman M, Lin HC, Chang HW, Wilson M, Lin MC. Normal tension glaucoma in patients with obstructive sleep apnea/hypopnea syndrome. J Glaucoma 2011; 20: 553-558.

9 Casas P, Ascaso FJ, Vicente E, Tejero-Garcés G, Adiego MI, Cristóbal JA. Retinal and optic nerve evaluation by optical coherence tomography in adults with obstructive sleep apnea-hypopnea syndrome (OSAHS). Graefes Arch Clin Exp Ophthalmol 2013; 251: 1625-1634.

10 Arden GB, Sidman RL, Arap W, Schlingemann RO. Spare the rod and spoil the eye. Br J Ophthalmol 2005; 89: 764-769.

11 Delaey C, van de Voorde J. Regulatory mechanisms in the retinal and choroidal circulation. Ophthalmic Res 2002; 32: 249-256.

12 Papst N, Demant E, Niemeyer G. Changes in PO2 induce retinal autoregulation in vitro. Graefes Arch Clin Exper Ophthalmol 1982; 219: 6-10.

13 West SD, Groves DC, Lipinski HJ, Nicoll DJ, Mason RH, Scanlon $\mathrm{PH}$ et al. The prevalence of retinopathy in men with type 2 diabetes and obstructive sleep apnoea. Diabet Med 2010; 27: 423-430.

14 Shiba T, Sato Y, Takahashi M. Relationship between proliferative diabetic retinopathy and sleep-disordered breathing. Nippon Ganka Gakkai Zasshi 2007; 111: 899-904.

15 Iber C, Ancoli-Israel S, Chesson Jr AL, Quan SF. The AASM manual for the scoring of sleep and associated events: rules, terminology and technical specifications. American Academy of Sleep Medicine: Westchester, IL, 2007.

16 Margolis R, Spaide RF. A pilot study of enhanced depth imaging optical coherence tomography of the choroid in normal eyes. Am J Ophthalmol 2009; 147: 811-815.

17 Manjunath V, Taha M, Fujimoto JG, Duker JS. Choroidal thickness in normal eyes measured using Cirrus HD optical coherence tomography. Am J Ophthalmol 2010; 150: 325-329.

18 Ding X, Li J, Zeng J, Ma W, Liu R, Li T et al. Choroidal thickness in healthy Chinese subjects. Invest Ophthalmol Vis Sci 2011; 52: 9555-9560.

19 Sogawa K, Nagaoka T, Takahashi A, Tanano I, Tani T, Ishibazawa A et al. Relationship between choroidal thickness and choroidal circulation in healthy young subjects. Am J Ophthalmol 2012; 153: 1129-1132.

20 Tan CS, Ouyang Y, Ruiz H, Sadda SR. Diurnal variation of choroidal thickness in normal, healthy subjects measured by spectral domain optical coherence tomography. Invest Ophthalmol Vis Sci 2012; 53: 261-266.

21 Bill A. Some aspects of the ocular circulation. (Friedenwald Lecture). Invest. Ophthalmol Vis Sci 1985; 26: 410-424.

22 Schrödl F, Schweigert M, Brehmer A, Neuhuber WL. Intrinsic neurons in the duck choroid are contacted by CGRP-immunoreactive nerve fibers: evidence for a local pre-central reflex arc in the eye. Exp Eye Res 2001; 72: 137-146.

23 Kergoat H, Marinier JA, Lovasik JV. Effects of transient mild systemic hypoxia on the pulsatile choroidal blood flow in healthy young human adults. Curr Eye Res 2005; 30: 465-470.

24 Geiser MH, Riva C, Dorner GT, Diermann U, Luksch A, Schmetterer L. Response of choroidal blood flow in the foveal region to hyperoxia and hyperoxia-hypercapnia. Curr Eye Res 2000; 21: 669-676.

25 Dioum EM, Chen R, Alexander MS, Zhang Q, Hogg RT, Gerard RD et al. Regulation of Hypoxia-inducible factor $2 \alpha$ signaling by the stress-responsive deacetylase Sirtuin 1 . Science 2009; 324: 1289-1293.

26 Liu J, Narasimhan P, Yu F, Chan PH. Neuroprotection by hypoxic preconditioning involves oxidative stress-mediated 
expression of hypoxia-inducible factor and erythropoietin. Stroke 2005; 36: 1264-1269.

27 Frayser R, Gray GW, Houston CS. Control of the retinal circulation at altitude. J Appl Physiol 1974; 37: 302-304.

28 Alm A. Ocular circulation. In: Hart WM (ed). Adler's Physiology of the Eye. Mosby-Year Book Inc.: St. Louis, MO, 1992, pp 198-227.

29 Imamura Y, Fujiwara T, Margolis R, Spaide RF. Enhanced depth imaging optical coherence tomography of the choroid in central serous chorioretinopathy. Retina 2009; 29 1469-1473.

30 Esmaeelpour M, Považay B, Hermann B, Hofer B, Kajic V, Hale SL et al. Mapping Choroidal and retinal thickness variation in type 2 diabetes using three-dimensional 1060-nm optical coherence tomography. Invest Ophthalmol Vis Sci 2011; 52: 5311-5316.

31 Mason RH, West SD, Kiire CA, Groves DC, Lipinski HJ, Jaycock A et al. High prevalence of sleep disordered breathing in patients with diabetic macular edema. Retina 2012; 32: 1791-1798.

32 Mason RH, Kiire CA, Groves DC, Lipinski HJ, Jaycock A, Winter BC et al. Visual improvement following continuous positive airway pressure therapy in diabetic subjects with clinically significant macular oedema and obstructive sleep apnoea: proof of principle study. Respiration 2012; 84: 275-282.

Supplementary Information accompanies this paper on Eye website (http://www.nature.com/eye) 